\title{
Li-Yorke chaos on one-dimensional map lattices
}

Lili Wei ${ }^{1}$ and Chenxing Zhou ${ }^{1 *}$

"Correspondence: mathcxzhou@163.com

${ }^{1}$ College of Mathematics, Changchun Normal University, Changchun, P.R. China

\begin{abstract}
In this paper, we consider a special, but important class of one-dimensional coupled map lattices, namely, in which the local dynamics including logistic map as a prototype possesses a snap-back repeller. For smaller coupling strengths, the existence of Li-Yorke scrambled set is proved.
\end{abstract}

MSC: 37E05; 37G10; 37G15

Keywords: Chaos; Logistic map; Snap-back repeller

\section{Introduction}

In spatially extended systems, spatiotemporal chaos, as a complex dynamical phenomenon, appears in a broad area of natural phenomena [1-10]. As a simple model for spatiotemporal chaos, one-dimensional map lattices $(O M L)$ have been proposed. These models contain coupled map lattices $(C M L)$, globally coupled map $(G M L)$ and open flow systems, etc. In this paper, we focus on the temporal chaos of a general form of $O M L$ :

$$
X_{n+1}=H\left(\mu, e, X_{n}\right),
$$

where

$$
H(\mu, e, X)=F(\mu, X)+e G(\mu, X), \quad F(\mu, X)=(f(\mu, x(1)), \ldots, f(\mu, x(L)))
$$

and $X=(x(1), \ldots, x(L)) \in R^{L}, L \geq 2, n \in Z_{+}$is the discrete time and $i(1 \leq i \leq L, L=$ systemsize) discrete space. The parameter $e$ and $G(\mu, X): R \times R^{L} \rightarrow R^{L}$, a $C^{r}$-map with $r \geq 1$, represent the strength of the coupling and the spatial interactions, respectively. Function $f: R \times R \rightarrow R$ is some $C^{r}$-map, with $r \geq 1$, satisfying

(H) For each parameter $\mu \in\left[\mu_{*}, \mu^{*}\right]\left(0 \prec \mu_{*} \prec \mu^{*}\right)$, there exist $r(\mu) \succ 0, x(\mu), \bar{x}(\mu)$ and integer $m(\mu) \geq 2$ such that

(i) $f(\mu, x(\mu))=x(\mu),\left|\frac{\partial f}{\partial x}(\mu, x)\right| \succ 1$ with $|x-x(\mu)| \leq r(\mu)$;

(ii) $0 \prec|\bar{x}(\mu)-x(\mu)| \prec r(\mu), f^{m(\mu)}(\mu, \bar{x}(\mu))=x(\mu)$ and $\frac{\partial f^{m(\mu)}}{\partial x}(\mu, \bar{x}(\mu)) \neq 0$.

By [11], $(H)$ is satisfied for $f(\mu, x)=\mu x(1-x)$, as a prototype for (1.1). The result below for $f:\left[\mu_{*}, \mu^{*}\right] \times \Omega \rightarrow R$, where $\Omega$ is an interval in $R$, is valid and it is only for notational purposes that the domain of definition of $f$ is taken to be $R \times R$. We note that (1.1) contains the following models:

(c) The Author(s) 2019. This article is distributed under the terms of the Creative Commons Attribution 4.0 International License (http://creativecommons.org/licenses/by/4.0/), which permits unrestricted use, distribution, and reproduction in any medium, provided you give appropriate credit to the original author(s) and the source, provide a link to the Creative Commons license, and indicate if changes were made. 
(I) If $g_{i}(\mu, X)=\frac{1}{2}[f(\mu, x(i-1))-2 f(\mu, x(i))+f(\mu, x(i+1))], 1 \leq i \leq L$, then $(1.1)$ becomes the most popular and well-studied model of CMLs:

$$
x_{n+1}(i)=f\left(\mu, x_{n}(i)\right)+\frac{1}{2}\left[f\left(\mu, x_{n}(i-1)\right)-2 f\left(\mu, x_{n}(i)\right)+f\left(\mu, x_{n}(i+1)\right)\right],
$$

which was originally introduced to model turbulent behavior as a synthesis of Landau's picture on turbulence [3] and Rössler's Hyperchaos [4].

(II) If $g_{i}(\mu, X)=\frac{1}{L} \sum_{j=1}^{L} f(\mu, x(j))-f(\mu, x(i)), 1 \leq i \leq L$, then (1.1) becomes a simple example of GLM, as a mean-field theory type extension of CML [5]:

$$
x_{n+1}(1-e)=(1-e) f\left(\mu, x_{n}(i)\right)+\frac{e}{L} \sum_{j=1}^{L} f\left(\mu, x_{n}(j)\right) .
$$

(III) If $g_{i}(\mu, X)=f(\mu, x(i-1))-f(\mu, x(i)), 1 \leq i \leq L$, then (1.1) becomes an open flow system (see also [6] for some other open flow models).

The importance of the models mentioned above is not restricted to dynamical systems. They are relevant to biological information processing [7], ecological models, evolutionary models [8], economics (e.g., stock market) and neural network [8]. The spatiotemporal chaos of these models has been studied extensively. Of particular interest is the existence of universality classes, like pattern selection, frozen random patterns, spatiotemporal intermittency and traveling waves. However, so far the results on temporal chaos are only numerical and by far incomplete (see [12]). The problem on the existence of Li-Yorke scrambled set (see $([11,13])$ of $(1.1)$ is completely open from the point of analytic studies. The purpose of the present paper is to prove the following result:

Theorem 1.1 If $(H)$ is satisfied, then there exists an $e_{*} \succ 0$ such that for any $(\mu, e) \in$ $\left[\mu_{*}, \mu^{*}\right] \times\left[-e_{*}, e_{*}\right]$, problem (1.1) is chaotic. That is, there exist:

(i) A positive integer $N(\mu, e)$ such that for each integer $p \geq N(\mu, e), H(\mu, e, \cdot)$ has a point of period $p$

(ii) A scrambled set of $H$, i.e., an uncountable set $S(\mu, e)$ containing no periodic points of $H(\mu, e, \cdot)$ such that

(a) $H(\mu, e, \cdot)[S(\mu, e)] \subset[S(\mu, e)]$,

(b) For every $X, Y \in S(\mu, e)$ with $X \neq Y, \lim _{\sup _{k \rightarrow \infty}}\left\|H^{k}(\mu, e, X)-H^{k}(\mu, e, Y)\right\| \succ 0$,

(c) For every $X \in S(\mu, e)$ and any periodic point $Y$ of $H(\mu, e, \cdot)$, we have

$$
\limsup _{k \rightarrow \infty}\left\|H^{k}(\mu, e, X)-H^{k}(\mu, e, Y)\right\| \succ 0
$$

(iii) An uncountable subset $S_{0}(\mu, e)$ of $S(\mu, e)$ such that for every $X, Y \in S_{0}(\mu, e)$, we have

$$
\liminf _{k \rightarrow \infty}\left\|H^{k}(\mu, e, X)-H^{k}(\mu, e, Y)\right\|=0
$$

where $\|X\|$ denotes the sup-norm of $X$ in $R^{L}:\|X\|=\max _{1 \leq j \leq L}|x(j)|$.

Our approach to the existence problem here is based on the well-known result of [11], Rouché Theorem and Implicit Function Theorem, etc. After introducing some basic definitions and theorems in Sect. 2, we will prove Theorem 1.1 in Sect. 3. Finally, some examples will be introduced to elucidate our result. 


\section{Some definitions and theorems}

Let $B_{r}(X)$ denote the closed ball in $R^{L}$ of radius $r$ centered at the point $X$ and let $B_{r}^{0}(X)$ be its interior. The following definition and theorems can be found in [11].

Definition 2.1 Let $\Phi: R^{L} \rightarrow R^{L}$ be differentiable on $B_{r}(Z)$. The point $Z \in R^{L}$ is an expanding fixed point of $\Phi$ in $B_{r}(Z)$, if $\Phi(Z)=Z$ and all eigenvalues of $D \Phi(Z)$ exceed 1 in norm for all $X \in B_{r}(Z)$; $Z$ is said to be a snap-back repeller of $\Phi$ if it is an expanding fixed point of $\Phi$ in $B_{r}(Z)$ and there exists a point $Z_{0} \in B_{r}^{0}(Z)$ with $Z_{0} \neq Z, \Phi^{M}\left(Z_{0}\right)=Z$, $\operatorname{det} D \Phi^{M}\left(Z_{0}\right) \neq 0$ for some positive integer.

Theorem 2.1 ([11, Theorem 3.1]) If $\Phi$ possesses a snap-back repeller, then system $X_{k+1}=$ $\Phi\left(X_{k}\right)$ is chaotic by means of Theorem 1.1.

The following Rouché Theorem on the continuity of the eigenvalues of a Jacobian matrix of parameters will be needed throughout the paper. For a proof, we refer to ([6], p. 248).

Theorem 2.2 (Rouché Theorem) Let $A$ be an open set in $C$, the set of complex numbers, $E$ a metric space, $\varphi$ a continuous complex valued function in $A \times E$, such that, for each $\alpha \in E$, $z \rightarrow \phi(z, \alpha)$ is analytic in $A$. Let $B$ be an open set of $A$, whose closure $\bar{B}$ in $C$ is compact and contained in $A$, and let $\alpha_{0} \in E$ be such that no zero of $\phi\left(z, \alpha_{0}\right)$ is on the boundary of $B$. Then there exists a neighborhood $W$ of $\alpha_{0}$ in $E$ such that:

(i) For any $\alpha \in W, \phi(z, \alpha)$ has no zeros on the boundary of $B$;

(ii) For any $\alpha \in W$, the sum of the orders of the zeros of $\phi(z, \alpha)$ belonging to $B$ is independent of $\alpha$.

The following Implicit Function Theorem is from [10, p. 3, Theorem 0.3].

Theorem 2.3 (Implicit Function Theorem) Let $U \subset R^{m} \times R^{n}$ be an open set and $\psi$ : $U \rightarrow R^{n}$ a $C^{r}$ map, for $r \geq 1$. Let $u_{0}=\left(x_{0}, y_{0}\right) \in U$ and $c=\psi\left(u_{0}\right)$. Suppose that the partial derivative with respect to the second variable, $D_{2} \psi\left(u_{0}\right): R^{n} \rightarrow R^{n}$, is an isomorphism. Then there exist open sets $V \subset R^{m}$ containing $x_{0}$ and $W \subset U$ containing $u_{0}$ such that, for each $x \in V$, there exists a unique $\xi(x) \in R^{n}$ with $(x, \xi(x)) \in W$ and its derivative is given by $d \xi(x)=\left[D_{2} \psi(x, \xi(x))\right]^{-1} \circ D_{1} \psi(x, \xi(x))$.

\section{Proof of Theorem 1.1}

Lemma 3.1 If $(H)$ is satisfied, then for any fixed $\mu_{0} \in\left[\mu_{*}, \mu^{*}\right]$, there exist $\delta^{\prime}\left(\mu_{0}\right) \succ 0$ and continuous $r_{0}(\mu, e), X_{0}(\mu, e)$ with $r_{0}\left(\mu_{0}, 0\right)=r\left(\mu_{0}\right), X_{0}\left(\mu_{0}, 0\right)=\left(x\left(\mu_{0}\right), \ldots, x\left(\mu_{0}\right)\right) \in R^{L} d e-$ fined on $\left[B_{\delta^{\prime}\left(\mu_{0}\right)}\left(\mu_{0}\right) \times B_{\delta^{\prime}\left(\mu_{0}\right)}(0)\right] \cap\left[\left[\mu_{*}, \mu^{*}\right] \times R\right]$ such that $H\left(\mu, e, X_{0}(\mu, e)\right)=X_{0}(\mu, e)$ and all eigenvalues of $D_{2} H(\mu, e, X)$ exceed 1 in norm for all $X \in B_{r_{0}(\mu, e)}\left(X_{0}(\mu, e)\right)$.

Proof Let $x_{0}=x\left(\mu_{0}\right), X_{0}=\left(x_{0}, \ldots, x_{0}\right) \in R^{L}, r_{0}=r\left(\mu_{0}\right)$. For any $x \in B_{r_{0}}\left(x_{0}\right),(H)$ gives us $\left|\frac{\partial f}{\partial x}\right| \succ 1$. Now, for any fixed $X=(x(1), \ldots, x(L)) \in B_{r_{0}}\left(X_{0}\right)$, we have

$$
D_{2} H\left(\mu_{0}, 0, X\right)=D_{2} F\left(\mu_{0}, X\right)
$$




$$
=\left|\begin{array}{cccc}
\frac{\partial f}{\partial x}\left(\mu_{0}, x(1)\right) & 0 & \ldots & 0 \\
0 & \frac{\partial f}{\partial x}\left(\mu_{0}, x(2)\right) & \ldots & 0 \\
\vdots & \vdots & \ddots & \vdots \\
0 & 0 & \ldots & \frac{\partial f}{\partial x}\left(\mu_{0}, x(L)\right)
\end{array}\right|
$$

thus all eigenvalues of $D_{2} H\left(\mu_{0}, 0, X\right)$ exceed 1 in norm.

Let $c_{1}=\frac{1}{2}\left[1+\min _{1 \leq j \leq L}\left|\frac{\partial f}{\partial x}\left(\mu_{0}, x(j)\right)\right|\right], c_{2}=\frac{1}{2}\left[1+\max _{1 \leq j \leq L}\left|\frac{\partial f}{\partial x}\left(\mu_{0}, x(j)\right)\right|\right]$, then $1 \prec c_{1} \prec c_{2}$.

Let $E=R \times R \times R^{L}, \alpha=\left(\mu_{0}, 0, X\right), A=C, B=\left\{\lambda \in C: c_{1} \prec|\lambda| \prec c_{2}\right\}$. Define continuous complex-valued function on $E \times C$ by

$$
\phi(\mu, e, Y, \lambda)=\operatorname{det}\left[\lambda I_{L}-D_{2} H(\mu, e, Y)\right]
$$

With $(\mu, e, Y, \lambda) \in\left[\mu_{*}, \mu^{*}\right] \times B_{r_{0}}(X) \times C$, where $I_{L}: R^{L} \rightarrow R^{L}$ is defined by $I_{L}(Y)=Y$, it is clear that no zero of $\phi\left(\mu_{0}, 0, X, \lambda\right)$ belonging to $B$. By Theorem 2.3 (Rouché Theorem), there exist $r_{X} \succ 0, \delta_{X} \succ 0$ so that for any $Y \in B_{r X}(X) \cap B_{r_{0}}(X), \mu \in B_{\delta X}\left(\mu_{0}\right) \cap\left[\mu_{*}, \mu^{*}\right]$ and $|e| \leq \delta_{X}$, all eigenvalues of $D_{2} H(\mu, e, Y)$ belong $B$, i.e., exceed 1 in norm. But $B_{r_{0}} \subset$ $U_{X \in B_{r_{0}}\left(X_{0}\right)} B_{r X}(X)$, there exist $X_{1}, \ldots, X_{q} \in B_{r_{0}}(X), r_{1}, \ldots, r_{q}$ and $\overline{\delta_{1}}, \ldots, \overline{\delta_{q}}$ such that

$$
B_{r_{0}}\left(X_{0}\right) \supset \bigcup_{j=1}^{q} B_{r j}\left(X_{J}\right)
$$

and for $Y \in B_{r j}\left(X_{j}\right) \cap B_{r_{0}}\left(X_{0}\right), \mu \in B_{\bar{\delta} j}\left(\mu_{0}\right) \cap\left[\mu_{*}, \mu^{*}\right]$ and $|e| \leq \overline{\delta_{j}}$, all eigenvalues of $D_{2} H(\mu, e, Y)$ exceed 1 in norm. Letting $\delta_{1}=\min _{1 \leq j \leq q} \bar{\delta}_{j}$, for any $X \in B_{r_{0}}\left(X_{0}\right), \mu \in B_{\delta_{1}}\left(\mu_{0}\right)$ and $|e| \leq \delta_{1}$, all eigenvalues of $D_{2} H(\mu, e, X)$ exceed 1 in norm.

On the other hand, from the fact that $f\left(\mu_{0}, x_{0}\right)=x_{0}$ we have that $H\left(\mu_{0}, 0, X_{0}\right)=X_{0}$. Letting $\psi(\mu, e, X)=H(\mu, e, H)-X$, we obtain $\psi\left(\mu_{0}, 0, X\right)=0$ and

$$
\operatorname{det} D_{2} \psi\left(\mu_{0}, 0, X_{0}\right)=\operatorname{det}\left[D_{2} F\left(\mu_{0}, X_{0}\right)-I_{L}\right]=\left[\frac{\partial f}{\partial x}\left(\mu_{0}, x_{0}\right)-1\right]^{L} \neq 0
$$

By Theorem 2.3 (Implicit Function Theorem), there exists $0 \prec \delta_{2} \leq \delta_{1}$ and continuous $X_{0}\left(\mu_{0}, e\right)$ defined on $B_{\delta_{2}}\left(\mu_{0}\right) \times B_{\delta_{2}}(0)$ such that $X_{0}\left(\mu_{0}, 0\right)=X_{0}, H\left(\mu, e, X_{0}(\mu, e)\right)=X_{0}(\mu, e)$. Choose $0 \prec \delta^{\prime}\left(\mu_{0}\right) \leq \delta_{2}$ so that $X_{0}(\mu, e) \in B_{r_{0}}^{0}\left(X_{0}\right), \mu \in B_{\delta^{\prime}\left(\mu_{0}\right)}\left(\mu_{0}\right), e \in B_{\delta^{\prime}\left(\mu_{0}\right)}(0)$. Therefore, we can choose $r(\mu, e)=\inf \left\{\left\|X_{0}(\mu, e)-Y\right\|:\left\|Y-X_{0}\right\|=r_{0}\right\}$, then $r\left(\mu_{0}, e\right) \succ 0$ is continuous with respect to $(\mu, e) \in B_{\delta^{\prime}(\mu)}\left(\mu_{0}\right) \times B_{\delta^{\prime}\left(\mu_{0}\right)}(0)$ and $r\left(\mu_{0}, 0\right)=r_{0}$. It is easy to see that $B_{r(\mu, e)}\left(X_{0}(\mu, e)\right) \subset B_{r_{0}}\left(X_{0}\right)$. Thus for any $(\mu, e) \in B_{\delta^{\prime}\left(\mu_{0}\right)} \times B_{\delta^{\prime}\left(\mu_{0}\right)}(0)$ and $Y \in B_{r(\mu, e)}\left(X_{0}(\mu, e)\right)$, all eigenvalues of $D_{2} H(\mu, e, Y)$ exceed $l$ in norm. The proof of Lemma 3.1 is complete.

Lemma 3.2 If $(H)$ is satisfied, for $\mu_{0}$ mentioned above, there exist $\delta\left(\mu_{0}\right)$ and continuous functions $X_{0}(\mu, e), r_{0}(\mu, e)$ and $\bar{X}_{0}(\mu, e)$ with $(\mu, e) \in B_{\delta\left(\mu_{0}\right)}\left(\mu_{0}\right) \times B_{\delta\left(\mu_{0}\right)}(0)$ such that

(i) $r_{0}\left(\mu_{0}, e\right)=r_{0}, X_{0}\left(\mu_{0}, 0\right)=X_{0}, \overline{X_{0}}\left(\mu_{0}, 0\right)=\left(\overline{x_{0}}\left(\mu_{0}\right), \ldots, \bar{x}\left(\mu_{0}\right)\right)$;

(ii) $H\left(\mu, e, X_{0}(\mu, e)\right)=X_{0}(\mu, e)$, all eigenvalues of $D_{2} H(\mu, e, X)$ exceed $l$ in norm for all $X \in B_{r_{0}(\mu, e)}\left(X_{0}(\mu, e)\right)$;

(iii) $\overline{X_{0}}(\mu, e) \in B_{r_{0}(\mu, e)}\left(X_{0}(\mu, e)\right), \overline{X_{0}}(\mu, e) \neq X_{0}(\mu, e), H^{m}\left(\mu, e, \overline{X_{0}}(\mu, e)\right)=X_{0}(\mu, e)$, $\operatorname{det}\left[D_{2} H^{m}\left(\mu, e, \overline{X_{0}}(\mu, e)\right)\right] \neq 0$,

where $m=m\left(\mu_{0}\right)$, i.e., $X_{0}(\mu, e)$ is a snap-back repeller of $H(\mu, e, \cdot)$. 
Proof Let $J(\mu, e, X)=H^{m}(\mu, e, X)-X_{0}(\mu, e)$, where $m=m\left(\mu_{0}\right),(\mu, e) \in B_{\delta^{\prime}\left(\mu_{0}\right)}\left(\mu_{0}\right) \times$ $B_{\delta^{\prime}\left(\mu_{0}\right)}(0)$ and $X_{0}(\mu, e)$ is defined as in Lemma 3.1. From this we get $J\left(\mu_{0}, 0, \overline{X_{0}}\right)=F^{m}\left(\mu_{0}\right.$, $\left.X_{0}\right)-X_{0}=0$ and

$$
\operatorname{det}\left[D_{2} J\left(\mu_{0}, 0, \overline{X_{0}}\right)\right]=\operatorname{det}\left[D_{2} F^{m}\left(\mu_{0}, X_{0}\right)\right]=\left[\frac{\partial f}{\partial x}\left(\mu_{0}, \bar{x}\left(\mu_{0}\right)\right)\right]^{L} .
$$

By $(H), \operatorname{det}\left[D_{2} J\left(\mu_{0}, 0, \bar{X}_{0}\right)\right] \neq 0$. Now Theorem 2.3 (Implicit Function Theorem) yields the existence of $0<\delta_{3} \leq \delta^{\prime}\left(\mu_{0}\right)$ and continuous function $X_{0}(\mu, e)$ on $B_{\delta_{3}}\left(\mu_{0}\right) \times B_{\delta_{3}}(0)$ such that $\bar{X}_{0}\left(\mu_{0}, 0\right)=\bar{X}_{0}, J\left(\mu, e, \bar{X}_{0}(\mu, e)\right)=0$, i.e., $H^{m}\left(\mu, e, \bar{X}_{0}(\mu, e)\right)=X_{0}(\mu, e)$. It is clear that $\bar{X}_{0}\left(\mu_{0}, 0\right)=\bar{X}_{0} \in B_{r_{0}}^{0}\left(X_{0}\right)$. Let $d_{0}=\left\|\bar{X}_{0}-X_{0}\right\|$, then $d_{0} \prec r_{0}$. We can chose $0 \prec \delta_{4} \leq \delta_{3}$ so that $\left\|\bar{X}_{0}-\bar{X}_{0}(\mu, e)\right\| \prec \frac{1}{3}\left(r_{0}-d_{0}\right),\left\|X_{0}-X_{0}(\mu, e)\right\| \prec \frac{1}{3}\left(r_{0}-d_{0}\right),\left|r(\mu, e)-r_{0}\right| \prec \frac{1}{3}\left(r_{0}-d_{0}\right)$, with $(\mu, e) \in B_{\delta_{4}}\left(\mu_{0}\right) \times B_{\delta_{4}}(0)$. It follows from this that

$$
\begin{aligned}
\left\|\bar{X}_{0}(\mu, e)-X_{0}(\mu, e)\right\| & \leq\left\|\bar{X}_{0}(\mu, e)-\bar{X}_{0}\right\|+\left\|\bar{X}_{0}-X_{0}\right\|+\left\|X_{0}-X_{0}(\mu, e)\right\| \\
& \leq \frac{1}{3}\left(r_{0}-d_{0}\right)+d_{0}+\frac{1}{3}\left(r_{0}-d_{0}\right)=r_{0}-\frac{1}{3}\left(r_{0}-d_{0}\right) \prec r(\mu, e),
\end{aligned}
$$

i.e., $X_{0}(\mu, e) \in B_{r_{0}(\mu, e)}\left(X_{0}(\mu, e)\right)$. Since $\operatorname{det}\left[D_{2} H^{m}\left(\mu_{0}, 0, \bar{X}_{0}\right)\right]=\left[\frac{\partial f^{m}}{\partial x}\left(\mu_{0}, \bar{x}\left(\mu_{0}\right)\right)\right]^{L} \neq 0$, we can chose $0 \prec \delta_{5} \leq \delta_{4}$ such that $\operatorname{det}\left[D_{2} H^{m}\left(\mu, e, \bar{X}_{0}(\mu, e)\right)\right] \neq 0$ with $(\mu, e) \in B_{\delta_{5}}\left(\mu_{0}\right) \times B_{\delta_{5}}(0)$.

The fact that $\bar{X}_{0}(\mu, e) \neq X_{0}$ gives us the existence of $0 \prec \delta\left(\mu_{0}\right) \leq \delta_{5}$ such that $\bar{X}_{0}(\mu, e) \neq$ $X_{0}(\mu, e)$ with $(\mu, e) \in B_{\delta\left(\mu_{0}\right)}\left(\mu_{0}\right) \times B_{\delta\left(\mu_{0}\right)}(0)$. The proof of Lemma 3.2 is complete.

Proof Lemma 3.2 yields that for any $\mu_{0} \in\left[\mu_{*}, \mu^{*}\right]$ there exists $\delta\left(\mu_{0}\right) \succ 0$ such that $X_{0}(\mu, e)$ is a snap-back repeller of $H(\mu, e, \cdot)$ with $(\mu, e) \in B_{\delta\left(\mu_{0}\right)} \times B_{\delta\left(\mu_{0}\right)}(0)$. Now, we note that $\left[\mu_{*}, \mu^{*}\right] \subset \bigcup_{\mu \in\left[\mu_{*}, \mu^{*}\right]} B_{\delta(\mu)}(\mu)$.

Thus there exist $\mu_{1}, \ldots, \mu_{q} \in\left[\mu_{*}, \mu^{*}\right]$ and $\delta_{1}^{*}, \ldots, \delta_{q}^{*}$ such that

$$
\left[\mu_{*}, \mu^{*}\right] \subset \bigcup_{j=1}^{q} B_{\delta^{*} j}\left(\mu_{j}\right)
$$

and, for each $1 \leq j \leq q$, there exist continuous $r_{j}(\mu, e) \succ 0, X_{j}(\mu, e)$ and $\bar{X}_{j}(\mu, e)$ such that

(i) $r_{j}\left(\mu_{j}, 0\right)=r\left(\mu_{j}\right), X_{j}\left(\mu_{j}, 0\right)=X\left(\mu_{j}\right)=\left(x\left(\mu_{j}\right), \ldots, x\left(\mu_{j}\right)\right)$,

$$
\bar{X}_{j}\left(\mu_{j}, 0\right)=\bar{X}\left(\mu_{j}\right)=\left(\bar{x}\left(\mu_{j}\right), \ldots, \bar{x}\left(\mu_{j}\right)\right) \text {; }
$$

(ii) $H\left(\mu, e, X_{j}(\mu, e)\right)=X_{j}(\mu, e)$ and all eigenvalues of $D_{2} H(\mu, e, X)$ exceed 1 in norm for all $X \in B_{r j(\mu, e)}\left(X_{j}(\mu, e)\right)$ with $(\mu, e) \in B_{\delta^{*} j}\left(\mu_{j}\right) \times B_{\delta^{*} j}(0)$;

(iii) $\bar{X}_{j}(\mu, e) \in B_{r j(\mu, e)}\left(X_{j}(\mu, e)\right), \bar{X}_{j}(\mu, e) \neq X_{j}(\mu, e)$ and $H^{m j}\left(\mu, e, \bar{X}_{j}(\mu, e)\right)=X_{j}(\mu, e)$,

$$
m_{j}=m\left(\mu_{j}\right), \operatorname{det}\left[D_{2} H^{m j}\left(\mu, e, \bar{X}_{j}(\mu, e)\right)\right] \neq 0 \text {, for }(\mu, e) \in B_{\delta^{*} j}\left(\mu_{j}\right) \times B_{\delta^{*} j}(0) \text {. }
$$

Letting $e_{*}=\min _{1 \leq j \leq q} \delta_{j}^{*}$, for each $(\mu, e) \in\left[\mu_{*}, \mu^{*}\right] \times\left[-e_{*}, e_{*}\right]$, there exists some $1 \leq j \leq q$ such that $(\mu, e) \in B_{\delta^{*} j}\left(\mu_{j}\right) \times B_{\delta^{*} j}(0)$, thus $X_{j}(\mu, e)$ is a snap-back repeller of $H(\mu, e, \cdot)$. Theorem 2.2 yields that (1.1) is chaotic. The proof of Theorem 1.1 is complete.

We conclude the paper with two examples.

Example 3.1 In system (1.1), we let $f(x)=\mu x(1-x)$. By [11, Example 4.1], we can find a snap-back repeller of $f$ for $\mu \succ 3.5$. Choose $3.5 \prec \mu_{*} \prec \mu^{*} \prec 4$, then Theorem 1.1 tells us 
that there exists $e_{*} \succ 0$ such that for any $(\mu, e) \in\left[\mu_{*}, \mu^{*}\right] \times\left[-e_{*}, e_{*}\right]$, with $f(x)=\mu x(1-x)$ is chaotic by means of Theorem 1.1.

Example 3.2 In system (1.1), let $f(x)=\mu x \exp (-x)$. By [11, Example 4.2], $f$ possesses a snap-back repeller for $v \succ 16.999$. Thus for any $16.999 \prec \mu_{*} \prec \mu^{*}$, Theorem 1.1 yields the existence of $e_{*} \succ 0$ such that (1.1) with $(\mu, e) \in\left[\mu_{*}, \mu^{*}\right] \times\left[-e_{*}, e_{*}\right]$ is chaotic by means of Theorem 1.1.

\section{Acknowledgements}

The authors are supported by the National Natural Science Foundation of China (Grant No. 11301038), The Natural Science Foundation of Jilin Province (Grant No. 20160101244JC).

\section{Funding}

Not applicable.

Availability of data and materials

Not applicable.

\section{Competing interests}

The authors declare that they have no competing interests.

\section{Authors' contributions}

All authors contributed equally to the manuscript. All authors read and approved the final manuscript.

\section{Publisher's Note}

Springer Nature remains neutral with regard to jurisdictional claims in published maps and institutional affiliations.

Received: 5 November 2018 Accepted: 15 January 2019 Published online: 07 May 2019

\section{References}

1. Chen, G., Tian, C.J., Shi, Y.M.: Stability and chaos in 2-D discrete systems. Chaos Solitons Fractals 25, $637-647$ (2005)

2. Kaneko, K.: Dynamical Problem in Soliton Systems. Springer, Berlin (1985)

3. Landau, L.D., Lifshtz, E.M.: Fluid Mechanics. Pergamon, Oxford (1959)

4. Rössler, O.E.: An equation for hyperchaos. Phys. Lett. A 71, 155-157 (1979)

5. Kaneko, K.: Clustering, coding, switching, hierarchical ordering, and control in a network of chaotic elements. Physica D 41, 137-172 (1990)

6. Willeboordse, F.H., Kaneko, K.: Pattern dynamics of a coupled map lattice for open flow. Physica D 86, 428-455 (1995)

7. Aref, H.: Integrable, chaotic turbulent vortex motion two-dimensional flows. Annu. Rev. Fluid Mech. 15, 345-389 (1983)

8. Eigen, M., Schuster, P.: The Hypercycle. Springer, Berlin (1979)

9. Cooper, L.N.: Proceedings of Nobel Symposium on Collective Properties of Physical Systems. Academic Press, New York (1973)

10. Willeboordse, F.H.: The spatial logistic map as a simple prototype for spatiotemporal chaos. Chaos 13, 533-540 (2003)

11. Marotto, F.R.: Snap-back repellers imply chaos in $\mathbb{R}^{N}$. J. Math. Anal. Appl. 63, 199-223 (1978)

12. Bunimovich, A.L.: Coupled map lattices: some topological and ergodic properties. Physica D 103, 1-17 (1997)

13. Li, T.Y., Yorke, J.A.: Period three implies chaos. Am. Math. Mon. 82, 985-992 (1975)

\section{Submit your manuscript to a SpringerOpen ${ }^{\circ}$ journal and benefit from:}

- Convenient online submission

- Rigorous peer review

- Open access: articles freely available online

- High visibility within the field

- Retaining the copyright to your article

Submit your next manuscript at $\gg$ springeropen.com 